\title{
Adiabatic quantum pumping in normal-metal-insulator-superconductor junctions in a monolayer of graphene
}

\author{
M. Alos-Palop and M. Blaauboer \\ Department of Quantum Nanoscience, Kavli Institute of Nanoscience, Delft University of Technology, \\ Lorentzweg 1, NL-2628 CJ Delft, The Netherlands \\ (Received 9 February 2011; revised manuscript received 5 May 2011; published 8 August 2011)
}

\begin{abstract}
We investigate adiabatic quantum pumping through a normal-metal-“insulator"-superconductor (NIS) junction in a monolayer of graphene. The pumped current is generated by periodic modulation of two gate voltages, applied to the insulating and superconducting regions, respectively. In the bilinear response regime and in the limit of a thin high insulating barrier, we find that the presence of the superconductor enhances the pumped current per mode by a factor of 4 at resonance. Compared to the pumped current in an analogous semiconductor NIS junction, the resonances have a $\pi / 2$ phase difference. We also predict experimentally distinguishable differences between the pumped current and the tunneling conductance in graphene NIS junctions.
\end{abstract}

DOI: 10.1103/PhysRevB.84.073402

PACS number(s): 72.80.Vp, 73.23.-b, 74.45.+c

Adiabatic pumping is a transport mechanism in meso- and nanoscale devices by which a finite dc current is generated in the absence of an applied bias by low-frequency periodic modulations of at least two system parameters (typically gate voltages or magnetic fields). ${ }^{1,2}$ In order for electrical transport to be adiabatic, the period of the oscillatory driving signals has to be much longer than the dwell time $\tau_{\text {dwell }}$ of the electrons in the system, $T=2 \pi \omega^{-1} \gg \tau_{\text {dwell }}$. Adiabatic quantum pumping ${ }^{3}$ refers to pumping in open systems in which quantum-mechanical interference of electron waves occurs. In the last decade, many different aspects of quantum pumping have been investigated in a diverse range of nanodevices, for example charge and spin pumping in quantum dots, ${ }^{4}$ the relation of quantum pumping to geometric (Berry) phases, ${ }^{5}$ and the role of electron-electron interactions. ${ }^{6}$ Quantum pumped currents have also been studied in hybrid systems consisting of normal-metal $(\mathrm{N})$ and superconducting $(\mathrm{S})$ parts, such as NS and SNS junctions. ${ }^{7-11}$ Recently, investigations of quantum pumping in graphene mono- and bilayers have appeared. ${ }^{12}$

In this Brief Report, we investigate adiabatic charge pumping through a normal-metal-“insulator"-superconductor (NIS) junction in graphene. The pumped current is generated by adiabatic variations of two gate voltages $U_{0}(t)$ and $V_{0}(t)$ which change, respectively, the Fermi level in the superconducting region and the height of the insulating tunnel barrier. The central question we aim to answer is what the effect of electron-hole (Andreev) reflection is on pumped charge currents in graphene. Using the scattering matrix formalism, we calculate the adiabatically pumped current at zero temperature in the linear response regime, i.e., for small variations of the pumping parameters $U_{0}$ and $V_{0}$, and we compare this with the pumped current in the absence of the superconducting lead. Our main result is that the presence of the superconducting lead enhances the pumped current per mode by a factor of 4 and the total pumped current by a factor of $3 \sqrt{2} / 2$ at the resonant tunneling condition. Off resonance, the pumped current is an order of magnitude smaller than the analogous current in a semiconductor NIS junction. We also find that whereas the conductance increases with $U_{0}$ for thin barriers, the pumped current decreases with $U_{0}$. This difference might be used to discriminate between conductance and pumped currents in graphene NIS junctions. In the last part of the Brief Report, we briefly comment on the pumped current in the so-called specular reflection regime (where $\Delta_{0} \geqslant E_{F}$ with $\Delta_{0}$ the superconductor gap and $E_{F}$ the Fermi energy). ${ }^{13}$

Consider the geometry depicted in Fig. 1. A ballistic sheet of graphene in the $(x, y)$ plane contains a potential barrier of height $V_{0}$ and length $d$ and a superconducting contact in the region $x \geqslant 0$. The barrier can be implemented by employing the electric field effect ${ }^{14,15}$ via a gate voltage and superconductivity can be induced in the region $x \geqslant 0$ via the proximity effect. We assume the potential step $V_{0}$ to be abrupt on both sides, which is justified close to the Dirac point where the Fermi wavelength $\lambda_{F} \gg d$ and which can be realized experimentally. ${ }^{15}$ The conductance $G(\mathrm{eV})$ through such a graphene NIS junction has recently been studied, both for potential barriers of finite length ${ }^{16}$ and in the limit of a thin barrier. ${ }^{17}$ Here we investigate the adiabatically pumped current through the latter junction. The pumped current is induced by periodic variations of $U_{0}(t)=U_{0}+\delta U_{0} \cos (\omega t)$ and $V_{0}(t)=$ $V_{0}+\delta V_{0} \cos (\omega t+\phi)$. The total pumped current $I$ into the

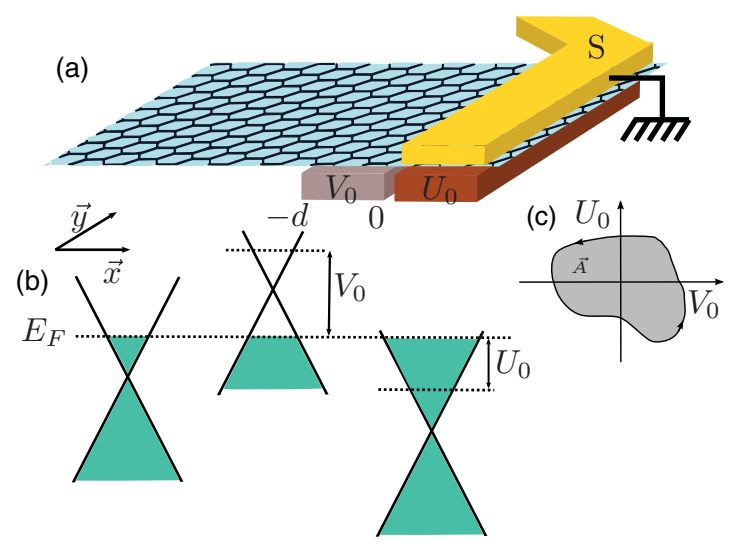

FIG. 1. (Color online) (a) Sketch of the graphene NIS junction. The variable gate voltage $V_{0}$ creates the insulating barrier (I) of length $d$ and the gate voltage $U_{0}$ is applied to the superconducting electrode (S). (b) Schematic of the energy levels in the three regions. (c) The area enclosed in the $\left(U_{0}, V_{0}\right)$ parameter space during one pumping cycle. 
normal lead (the left contact in Fig. 1) can be expressed as an integral over the area $A$ that is enclosed in $\left(U_{0}, V_{0}\right)$ parameter space during one period, and is given by the scattering matrix expression $^{8}$

$$
\begin{aligned}
I \equiv I_{N} & =\frac{\omega e}{2 \pi^{2}} \int_{A} d U_{0} d V_{0} \sum_{\alpha, \beta \in N} \Pi_{\alpha \beta}\left(U_{0}, V_{0}\right) \\
& \approx \frac{\omega e}{2 \pi} \delta U_{0} \delta V_{0} \sin \phi \sum_{\alpha, \beta \in N} \Pi_{\alpha \beta}\left(U_{0}, V_{0}\right)
\end{aligned}
$$

with

$$
\Pi_{\alpha \beta}\left(U_{0}, V_{0}\right) \equiv \operatorname{Im}\left(\frac{\partial S_{\alpha \beta}^{e e \star}}{\partial U_{0}} \frac{\partial S_{\alpha \beta}^{e e}}{\partial V_{0}}-\frac{\partial S_{\alpha \beta}^{h e \star}}{\partial U_{0}} \frac{\partial S_{\alpha \beta}^{h e}}{\partial V_{0}}\right) .
$$

Equation (2) is valid in the bilinear response regime where $\delta U_{0} \ll U_{0}+E_{F}$ and $\delta V_{0} \ll V_{0}+E_{F}$ and the integral in Eq. (1) becomes independent of the pumping contour. The indices $\alpha$ and $\beta$ sum over all modes in the normal lead and $S$ denotes the Landauer-Büttiker scattering matrix whose elements $S_{\alpha \beta, n m}^{h e}$ describe the scattering of an electron in mode $m$ in lead $\beta$ to a hole in mode $n$ in lead $\alpha$.

The low-energy excitations in the NIS junction close to the Dirac point $K\left(K^{\prime}\right)$ are described by the $4 \times 4$ DiracBogoliubov-de Gennes Hamiltonian ${ }^{13}$

$$
\mathcal{H}=\left(\begin{array}{cc}
\mathcal{H}_{a}-E_{F}+U(x) & \Delta(x) \\
\Delta^{*}(x) & E_{F}-U(x)-\mathcal{H}_{a}
\end{array}\right)
$$

with $\mathcal{H}_{a}=-i \hbar v_{F}\left[\sigma_{x} \partial_{x}+\operatorname{sgn}(a) \sigma_{y} \partial_{y}\right]$, where $\operatorname{sgn}(a)$ is \pm for $a=K\left(K^{\prime}\right), v_{F}$ denotes the Fermi velocity of the quasiparticles, and the potential $U(x)=-U_{0} \theta(x)+V_{0} \theta(-x) \theta(x+d)$. The pair potential $\Delta(x)$ which couples the electron and hole excitations has the form $\Delta_{0} e^{i \phi}$ and we assume that $\left(E_{F}+\right.$ $\left.U_{0}\right) \gg \Delta_{0}$, the mean-field condition for superconductivity. Two regimes can be distinguished: $E_{F} \gg \Delta_{0}$ where the usual retro Andreev reflection dominates, and $E_{F} \leqslant \Delta_{0}$ so $U_{0} \gg \Delta_{0}$, where specular Andreev reflection dominates. ${ }^{13}$ Our analysis focuses on the retro reflection regime unless we explicitly mention that we study the specular reflection regime. The Hamiltonian (4) acts on the four-component wave function $\psi_{a}=\left(\psi_{A a}, \psi_{B a}, \psi_{A \bar{a}}^{*},-\psi_{B \bar{a}}^{*}\right)$, where $A$ and $B$ denote the two nonequivalent sites of the graphene unit lattice and $\bar{a}=K^{\prime}(K)$ for $a=K\left(K^{\prime}\right)$. Following Ref. 17, we also introduce the dimensionless barrier strength $\chi=d V_{0} /\left(\hbar v_{F}\right)$ which allows us to consider the limit of a thin barrier where $V_{0} \rightarrow \infty$ and $d \rightarrow 0$ such that $\chi$ remains finite.

After applying continuity of the wave functions at the boundaries $x=-d$ and $x=0$, one obtains the reflection and transmission coefficients of the NIS junction; see Eqs. (9) in Ref. 17. At the Dirac point $\epsilon=0$, where $\epsilon$ denotes the energy of the electrons measured from the Fermi level, ${ }^{18}$ and defining $\delta=E_{F} /\left(E_{F}+U_{0}\right)$, the derivatives of the coefficients for normal and Andreev reflection, $r$ and $r_{A}$, with respect to the gate voltages $U_{0}$ and $V_{0}$ are given by $\partial r_{(A)} / \partial U_{0}=\left(\partial r_{(A)} / \partial \delta\right)\left(\partial \delta / \partial U_{0}\right)$ and $\partial r_{(A)} / \partial V_{0}=$ $\left(\partial r_{(A)} / \partial \chi\right)\left(\partial \chi / \partial V_{0}\right)$ with

$$
\begin{gathered}
\frac{\partial r}{\partial \chi}=\frac{2 e^{i \alpha} \delta \sin \alpha \cos \alpha\left(\cos 2 \chi+\delta \sin ^{2} \alpha-i \cos \alpha \sin 2 \chi\right)}{\left(1+\delta \sin ^{2} \alpha \cos 2 \chi\right)^{2}}, \\
\frac{\partial r^{*}}{\partial \delta}=\frac{-i e^{-i \alpha} \sin \alpha \cos \alpha(\cos 2 \chi \cos \alpha+i \sin 2 \chi)}{\left(1+\delta \sin ^{2} \alpha \cos 2 \chi\right)^{2}},
\end{gathered}
$$

$$
\begin{gathered}
\frac{\partial r_{A}}{\partial \chi}=\frac{-2 i e^{-i \phi} \delta \sin ^{2} \alpha \cos \alpha \sqrt{1-\delta^{2} \sin ^{2} \alpha} \sin 2 \chi}{\left(1+\delta \sin ^{2} \alpha \cos 2 \chi\right)^{2}}, \\
\frac{\partial r_{A}^{*}}{\partial \delta}=\frac{-i e^{i \phi} \sin ^{2} \alpha \cos \alpha(\delta+\cos 2 \chi)}{\sqrt{1-\delta^{2} \sin ^{2} \alpha}\left(1+\delta \sin ^{2} \alpha \cos 2 \chi\right)^{2}} .
\end{gathered}
$$

Here $\alpha$ is the angle of incidence of the electron. Substituting Eq. (5) into Eq. (2) and integrating over the angle of incidence yields the pumped current at $\epsilon=0$,

$$
\begin{gathered}
I_{g}^{N I S}=I_{g, 0} \frac{\delta^{3}}{\pi} \int_{0}^{\pi / 2} d \alpha \frac{\sin ^{2} \alpha \cos ^{4} \alpha}{\left(1+\delta \sin ^{2} \alpha \cos 2 \chi\right)^{3}} \\
\stackrel{U_{0}=0}{=} I_{g, 0} \frac{4 \cos ^{4} \chi-12 \cos ^{2} \chi+8 \sqrt{2}|\cos \chi|-3}{16 \sqrt{2}|\cos \chi|\left(2 \cos ^{2} \chi-1\right)^{3}},
\end{gathered}
$$

where $I_{g, 0} \equiv \omega e \frac{d}{\hbar v_{F} E_{F}} \delta U_{0} \delta V_{0} \sin \phi$. Equation (7) is valid for $U_{0}=0$. Note from Eq. (6) that there is no contribution to $I_{g}^{\text {NIS }}$ from normally incident electrons with $\alpha=0$. This is a display of the Klein tunneling effect where the Andreev reflection coefficient $\left|r_{A}(\alpha=0)\right|=1$ independent of $\chi$ and $U_{0}$.

Figure 2(a) shows the pumped current, Eq. (7), as a function of $\chi$. Notice that $I_{g}^{N I S}$, just like the conductance in this system, ${ }^{17}$ is periodic in $\chi$ with a period $\pi$. We see that $I_{g}^{N I S}$ reaches maximum values when $V_{0} d=(n+1 / 2) \pi \hbar v_{F}$, where $n$ is an integer. This is the condition for resonant transmission (i.e., $r=0$ ) when the conductance of the system reaches a sharply peaked maximum. ${ }^{17}$ Due to the sharp changes in the derivative of $r$ the pumped current diverges at this point. Expanding Eq. (7) with respect to $\chi$ around $\chi=0$, we find that $I_{g}^{N I S}$ scales as

$$
I_{g}^{N I S} / I_{g, 0} \sim a_{0}+a_{1} \chi^{2},
$$

where $a_{0}=\frac{16-11 \sqrt{2}}{32} \approx 0.014$ and $a_{1}=3\left(1-\frac{45}{32 \sqrt{2}}\right) \approx 0.017$. The pumped current thus increases with the barrier height close to $\chi=0$, which is another manifestation of the Klein paradox. Next, we analyze the behavior of the current with respect to $U_{0}$. We expand Eq. (6) with respect to $U_{0}$ around $U_{0}=0$ at $\chi=0$, which results in

$$
I_{g}^{N I S} / I_{g, 0} \sim b_{0}-b_{1} U_{0},
$$

where $b_{0}=a_{0}$ and $b_{1}=\frac{3 \sqrt{2}}{128} \approx 0.033$. Equation (9) shows that the pumped current decreases with increasing $U_{0}$.
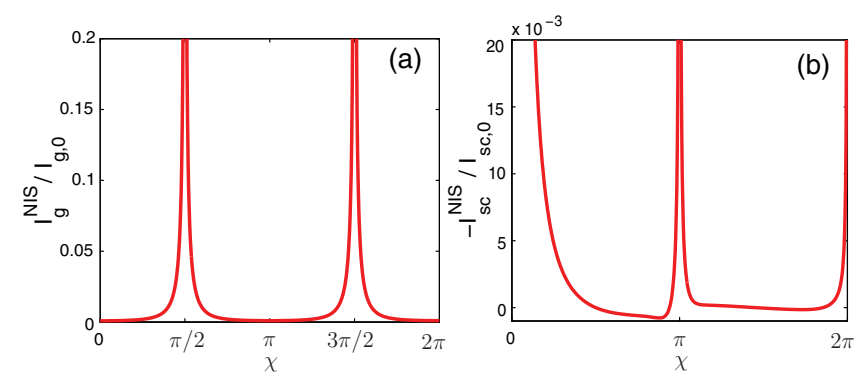

FIG. 2. (Color online) (a) The pumped current $I_{g}^{N I S}$ [Eq. (7)] as a function of the potential barrier strength $\chi$ in a graphene (g) NIS junction. (b) The analogous pumped current $I_{s c}^{N I S}$ [Eq. (10)] in a semiconductor (sc) NIS junction, where $\sqrt{2 m E_{F}} d / \hbar=10$. 
Figure 2(b) shows the corresponding pumped current $I_{s c}^{N I S}$ in a semiconductor NIS junction. We model the insulating region again as a barrier of height $V_{0}$ and width $d$, and define the dimensionless barrier strength $\chi=\sqrt{2 m V_{0}} d / \hbar$. Solving the Bogoliubov-de Gennes equation, matching the wave function and its derivative at the boundaries, obtaining $r$ and $r_{A}$, and calculating the derivatives with respect to the gate voltages $V_{0}$ and $U_{0}$ yields the pumped current (for $\epsilon=0$ and for $U_{0}=0$ ):

$$
\begin{aligned}
I_{s c}^{N I S}= & I_{s c, 0} \int_{0}^{\pi / 2} d \alpha \cos \alpha k^{3} d^{3} \chi^{3} \\
& \times \frac{\left[2 \chi\left(k^{2} d^{2}-\chi^{2}\right)-\left(k^{2} d^{2}+\chi^{2}\right) \sin 2 \chi\right]}{\left[2 k^{2} d^{2} \chi^{2} \cos ^{2} \chi+\left(k^{4} d^{4}+\chi^{4}\right) \sin ^{2} \chi\right]^{3}},
\end{aligned}
$$

where $\quad I_{s c, 0} \equiv(\omega e / \pi)\left(2 m^{2} d^{4} / \hbar^{4}\right) \delta U_{0} \delta V_{0} \sin \phi \quad$ and $k \equiv$ $\left(\sqrt{2 m E_{F}} / \hbar\right) \cos \alpha$. Equations (7) and (10) are the main results of this Brief Report. From Eq. (10) we notice that, in contrast to graphene, the normally incident electrons in the semiconductor junction do contribute to the pumped current, illustrating the absence of Klein tunneling in a semiconductor NIS junction. Figure 2(b) displays $I_{s c}^{N I S}$ as a function of the barrier strength $\chi$. We observe that $I_{s c}^{N I S}$ also oscillates as a function of $\chi$ with a period of $\pi$. However, the maxima at resonant transmission occur when $\chi=n \pi$ and are thus shifted by $\pi / 2$ with respect to the maxima of $I_{g}^{N I S}$. Notice that $I_{s c}^{N I S}$ and $I_{g}^{N I S}$ mostly have opposite signs and that $I_{s c}^{N I S}$ switches sign at several points. In addition, the pumped current in a semiconductor NIS junction is roughly one order of magnitude larger than in graphene. ${ }^{19}$

An important question arising when thinking about experimental detection of pumped currents is how to distinguish them from the conductance $G$ in the system. In order to answer this question we explicitly compare both quantities. The conductance of the NIS junction in graphene was considered in Ref. 17 and is given by, at $\epsilon=0$ and $U_{0}=0$,

$G_{g}^{N I S}=G_{0} \frac{\sqrt{A}(A+3)+\left(A^{2}-2 A-3\right) \arctan (\sqrt{A})}{A^{5 / 2}}$,

where $G_{0} \equiv \frac{4 e^{2}}{h} \frac{E_{F} W}{\pi \hbar v_{F}}$ with $W$ the width of the sample and $A \equiv \cos 2 \chi$. Expansion of $G_{g}^{N I S}$ for small $\chi$ around $\chi=0$ at $U_{0}=0$ and for small $U_{0}$ around $U_{0}=0$ at $\chi=0$ yields, respectively,

$$
\begin{gathered}
G_{g}^{N I S} / G_{0} \sim(4-\pi)+(16-5 \pi) \chi^{2}, \\
G_{g}^{N I S} / G_{0} \sim(4-\pi)+(2-\pi / 2) U_{0} / E_{F} .
\end{gathered}
$$

We find that $G_{g}^{N I S}=(4-\pi) G_{0}$ for $U_{0}=0$ and for $\chi=0$, somewhat below the ballistic value $G_{0}$ due to mismatch in Fermi wavelength in the normal and superconducting leads, as mentioned earlier. ${ }^{13}$ Comparing the scaling behavior of $G_{g}^{N I S}$ and $I_{g}^{N I S}$ as a function of $\chi$, Eqs. (8) and (12), we find that both transport quantities increase with increasing $\chi$. However, when comparing Eqs. (9) and (13) we see that whereas the pumped current $I_{g}^{N I S}$ decreases with increasing $U_{0}$, the conductance $G_{g}^{N I S}$ increases with increasing $U_{0}$. Intuitively, switching on $U_{0}$ increases the Fermi level mismatch, which increases the conductance ${ }^{20}$ but decreases the pumped current. This difference can be used to discriminate the pumped current from the conductance in an actual experiment.
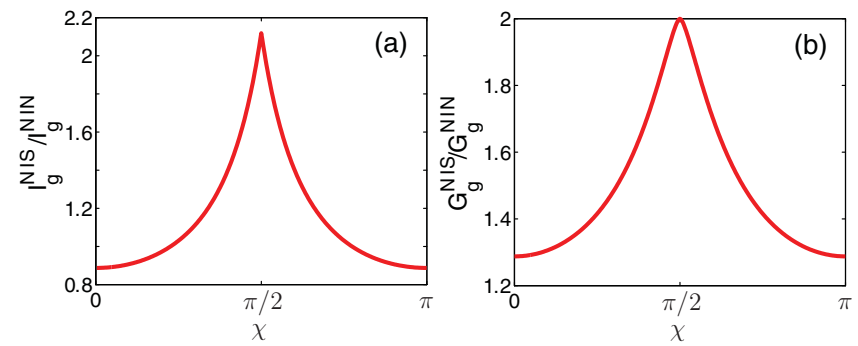

FIG. 3. (Color online) Ratio of the pumped current (left) and the conductance (right) of the NIS junction and the NIN junction in graphene versus $\chi$ for $\epsilon=0$ and $U_{0}=0$.

We now investigate the influence of the superconducting lead on the pumped current by comparing $I_{g}^{N I S}$ with the pumped current $I_{g}^{N I N}$ through an entirely normal NIN junction in graphene. At the Dirac point $\epsilon=0$ and for $U_{0}=0$ the latter is given by

$$
I_{g}^{N I N}=I_{g, 0} \frac{(1-|\cos \chi|)^{2}}{16|\cos \chi| \sin ^{4} \chi} .
$$

Similarly, the conductance through a NIN junction is given by

$$
G_{g}^{N I N}=G_{0} \frac{\sin \chi-\cos ^{2} \chi \operatorname{arctanh}(\sin \chi)}{\sin ^{3} \chi} .
$$

Figure 3 shows the ratios $I_{g}^{N I S} / I_{g}^{N I N}$ and $G_{g}^{N I S} / G_{g}^{N I N}$ versus $\chi$.In both cases, the superconducting lead enhances the transport, reaching a maximum at $\chi=\pi / 2$. For the conductance this maximum enhancement is 2 due to the contribution of the holes, ${ }^{21}$ while the enhancement of the pumped current rises from $2(16-11 \sqrt{2}) \approx 0.89$ at $\chi=0$ to a factor of $3 \sqrt{2} / 2 \approx$ 2.12 at $\chi=\pi / 2$. However, when comparing $I_{g}^{N I S}$ [Eq. (6)] and $I_{g}^{N I N}$ per mode at $\chi=\pi / 2$, i.e., before integration over $\alpha$, we see that the superconducting lead enhances the pumped current of each mode by a factor of 4 . This last result is due to both the holes, which contribute a factor of 2 , and the asymmetry of the NIS junction with respect to injection of charge carriers, which contributes another factor of $2 .{ }^{8}$

At this point we briefly mention the behavior of the pumped current as a function of an applied bias voltage $\mathrm{eV}$ both in the normal (retro) and in the specular Andreev reflection regime. ${ }^{22}$ For bias voltages below the gap $e V \leqslant \Delta_{0}$, the pumped current $I_{g}^{N I S}$ in the retro reflection regime decreases from a finite value at $e V=0$ [see Eq. (7)] to zero at $e V=\Delta_{0}$. At $e V=\Delta_{0}$, the particles are fully Andreev reflected $\left(\left|r_{A}\right|=1\right.$ independent of $\chi$ and $\left.U_{0}\right)$ and therefore $I_{g}^{N I S}=0$. In the specular reflection regime, ${ }^{13}$ where $E_{F} \leqslant \Delta_{0}$ and $U_{0} \gg E_{F}$, the pumped current exhibits different behavior. First, the pumped current, just like the conductance, ${ }^{17}$ is insensitive to $\chi$ for energies below the gap. The large mismatch in Fermi energies of the normal and the superconducting leads already acts as a barrier, and as a result the addition of another barrier is irrelevant, explaining this behavior. Furthermore, the pumped current $I_{g}^{N I S}$ is zero for bias voltages equal to the Fermi level $e V=E_{F}$, due to the absence of Andreev reflection, ${ }^{13}$ and also for $e V=\Delta_{0}$; see the discussion above. As a final remark, the pumped current is several orders of magnitudes smaller than in the retro reflection regime. 
Finally, we briefly comment on possibilities for experimental observation of our predictions. Experiments with superconducting electrodes on top of graphene in which multiple Andreev reflections were observed have already been carried out. ${ }^{23,24}$ From these experiments we can estimate the order of magnitude of the pumped current. Some typical parameters are $\omega /(2 \pi)=5 \mathrm{GHz}, E_{F}=80 \mathrm{meV}, v_{F}=10^{6} \mathrm{~m} / \mathrm{s}$, and barrier width $d=10-20 \mathrm{~nm} \cdot{ }^{15,25}$ For gate voltages on the order of 10 $\mathrm{meV}$, the pumped current is on the order of $10 \mathrm{fA}$ far from the resonant tunneling condition, going up to $0.1-1 \mathrm{pA}$ or higher close to resonance.

In conclusion, we have investigated adiabatic quantum pumping in a graphene NIS junction, which is generated by periodic modulation of the insulating barrier $V_{0}$ and the Fermi level on the superconductor side. We have demonstrated that the presence of the superconducting lead can enhance the pumped current per mode by a factor of 4 (at resonance) and suggested experimentally observable differences between the conductance and the pumped current in this system.

Recently we became aware of another work on quantum pumping in graphene in which a junction with two superconducting leads is considered. ${ }^{26}$

This work has been supported by the Netherlands Organization for Scientific Research (NWO/FOM).
${ }^{1}$ M. Büttiker, H. Thomas, and A. Prêtre, Z. Phys. B 94, 133 (1994).

${ }^{2}$ P. W. Brouwer, Phys. Rev. B 58, 10135(R) (1998).

${ }^{3}$ B. Spivak, F. Zhou, and M. T. Beal Monod, Phys. Rev. B 51, 13226 (1995).

${ }^{4}$ M. Switkes, C. M. Marcus, K. Campman, and A. D. Gossard, Science 283, 1905 (1999); E. R. Mucciolo, C. Chamon, and C. M. Marcus, Phys. Rev. Lett. 89, 146802 (2002); P. Sharma and P. W. Brouwer, ibid. 91, 166801 (2003); S. K. Watson, R. M. Potok, C. M. Marcus, and V. Umansky, ibid. 91, 258301 (2003).

${ }^{5}$ J. E. Avron, A. Elgart, G. M. Graf, and L. Sadun, Phys. Rev. B 62, 10618(R) (2000).

${ }^{6}$ J. Splettstoesser, M. Governale, J. König, and R. Fazio, Phys. Rev. Lett. 95, 246803 (2005); E. Sela and Y. Oreg, ibid. 96, 166802 (2006); F. Reckermann, J. Splettstoesser, and M. R. Wegewijs, ibid. 104, 226803 (2010).

${ }^{7}$ J. Wang, Y. Wei, B. Wang, and H. Guo, Appl. Phys. Lett. 79, 3977 (2001).

${ }^{8}$ M. Blaauboer, Phys. Rev. B 65, 235318 (2002).

${ }^{9}$ F. Taddei, M. Governale, and R. Fazio, Phys. Rev. B 70, 052510 (2004).

${ }^{10}$ M. Governale, F. Taddei, R. Fazio, and F. W. J. Hekking, Phys. Rev. Lett. 95, 256801 (2005).

${ }^{11}$ J. Splettstoesser, M. Governale, J. König, F. Taddei, and R. Fazio, Phys. Rev. B 75, 235302 (2007).

${ }^{12}$ E. Prada, P. San-Jose, and H. Schomerus, Phys. Rev. B 80, 245414 (2009); R. Zhu and H. Chen, Appl. Phys. Lett. 95, 122111 (2009); E. Prada, P. San-Jose, and H. Schomerus, Solid State Commun. 151, 1065 (2011); G. M. M. Wakker and M. Blaauboer, Phys. Rev. B 82, 205432 (2010).

${ }^{13}$ C. W. J. Beenakker, Phys. Rev. Lett. 97, 067007 (2006).

${ }^{14}$ K. S. Novoselov, A. K. Geim, S. V. Morozov, D. Jiang, Y. Zhang, S. V. Dubonos, I. V. Grigorieva, and A. A. Firsov, Science 306, 666 (2004).
${ }^{15}$ M. I. Katsnelson, K. S. Novoselov, and A. K. Geim, Nature Phys. 2, 620 (2006).

${ }^{16}$ S. Bhattacharjee, M. Maiti, and K. Sengupta, Phys. Rev. B 76, 184514 (2007)

${ }^{17}$ S. Bhattacharjee and K. Sengupta, Phys. Rev. Lett. 97, 217001 (2006).

${ }^{18}$ We remark that $\epsilon=0$ is the point of highest interest for observing quantum pumping, since it corresponds to the situation in which no bias voltage is applied.

${ }^{19}$ Here we used that $I_{s c}^{N I S}=I_{g}^{N I S}\left(2 m E_{F}\right)^{3 / 2} d^{3} /\left(\pi \hbar^{3}\right)$.

${ }^{20}$ See also Ref. 13, where it was assumed that $U_{0} \gg E_{F}$. The conductance is then given by $G=(4 / 3) G_{0}$ at zero bias $e V=0$ and decreases to the value $G=(4-\pi) G_{0}$ for $e V \gg \Delta_{0}$, in which case the Fermi level mismatch is minimal.

${ }^{21}$ C. W. J. Beenakker, in Mesoscopic Quantum Physics, edited by E. Akkermans, G. Montambaux, J.-L. Pichard, and J. Zinn-Justin (North-Holland, Amsterdam, 1995).

${ }^{22} \mathrm{~A}$ more extensive analysis is under way and will be presented elsewhere.

${ }^{23}$ H. B. Heersche, P. Jarillo-Herrero, J. B. Oostinga, L. M. K. Vandersypen, and A. F. Morpurgo, Nature (London) 446, 56 (2007).

${ }^{24}$ F. Miao, M. S. Wijeratne, Y. Zhang, U. C. Coskun, W. Bao, and C. N. Lau, Science 14, 1530 (2007).

${ }^{25}$ K. S. Novoselov, A. K. Geim, S. V. Morozov, D. Jiang, M. I. Katsnelson, I. V. Grigorieva, S. V. Dubonos, and A. A. Firsov, Nature (London) 438, 197 (2005); Y. Zhang, Y.-W. Tan, H. L. Stormer, and P. Kim, ibid. 438, 201 (2005); K. S. Novoselov, E. McCann, S. V. Morozov, V. I. Fal'ko, M. I. Katsnelson, U. Zeitler, D. Jiang, F. Schedin, and A. K. Geim, Nature Phys. 2, 177 (2006).

${ }^{26}$ A. Kundu, S. Rao, and A. Saha, Phys. Rev. B 83, 165451 (2011). 